RESEARCH ARTICLE

\title{
The motive behind the demand for cryptocurrencies: Theoretical and empirical analysis of the Bitcoin price
}

\author{
Mengshi Zhang ${ }^{1}$ Daniel L. Jia ${ }^{2^{*}}$ \\ ${ }^{1}$ Graduate School of Chinese Academy of Social Sciences \& CNCC, Beijing 102488, China \\ ${ }^{2}$ Development Research Center of the State Council (DRC), Beijing 100010, China
}

\section{Check for updates}

Correspondence to: Daniel L. Jia, Development Research Center of the State Council (DRC), Beijing 100010, China; E-mail: daniellukuijia@126.com

Received: August 22, 2021;

Accepted: October 24, 2021;

Published: October 28, 2021.

Citation: Zhang M and Jia DL. The motive behind the demand for cryptocurrencies: Theoretical and empirical analysis of the Bitcoin price. Front Manage Bus, 2021, 2(1): 101-117.

https://doi.org/10.25082/FMB.2021.01.005

Copyright: (c) 2021 Mengshi Zhang and Daniel L. Jia. This is an open access article distributed under the terms of the Creative Commons Attribution License, which permits unrestricted use, distribution, and reproduction in any medium, provided the original author and source are credited.

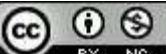

\begin{abstract}
The blockchain technology and cryptocurrency are now in the centre of the financial market. The raise of the cryptocurrencies represented by Bitcoin have attracted a large group of scholars to analyze the underlying dynamics of their price fluctuations. Intensive debate emerged on the intrinsic features of Bitcoin. In theoretical analysis, we developed the principle of monetary convention to define the concept of monetary consensus, capturing the nature of monetary system, and categorize it into three types: traditional, algorithm and hybrid. Based on the Wavelet Coherence Analysis, we try to analyze Bitcoin price dynamics in both time and frequency domains, comparing Bitcoin with financial assets, economic and financial indexes, and other cryptocurrencies.
\end{abstract}

Keywords: cryptocurrency, price dynamics, wavelet coherence

\section{Introduction}

Digital currency and digitization of currency are two intertwined but different concepts. The former is a form of money, while the latter is a trend of money development with the development of modern computer technology and the evolution of financial innovation. In such sense, digital currency is not only the carrier of currency digitization, but also the result of currency digitization. We are no stranger to traditional currency digitization and digital currency (or electronic currency, EC). In daily life, transactions and transfers using digital currency are carried out all the time. Different from the traditional currency digitization based on the central bank-commercial bank account system (When studying the modern payment system, we also need to include the third-party payment institutions. However, this change is still a limited expansion of the traditional central bank commercial banking system. Both central banks, commercial banks and third-party payment institutions use centralized methods to support customer transactions. This is essentially different from the decentralized monetary system represented by digital cryptocurrency.), cryptocurrencies represented by Bitcoin is committed to establishing a decentralized and super sovereign currency system based on blockchain technology and consensus principle, aiming to achieve safe and convenient P2P payment Transactions are completed through smart contracts, and the ledger information of transactions is saved in the blockchain without being stored in a central institution (such as the current commercial bank database and third-party payment database). The customer's personal and transactional information are effectively isolated, so the protection of user's personal information is realized while solving the double-spending problem. In such a cryptocurrency system, the main body of currency issuance will no longer be the banking system dominated by the central bank, and the support of national credit is no longer needed behind currency issuance. This subversive concept of tradition has aroused fierce discussion from the beginning.

In the past few years, the expansion of cryptocurrencies represented by Bitcoin has exceeded most people's expectations. Bitcoin has quickly become a priority research object for market participants and research institutions. Moreover, with the rapid growth of Bitcoin price, more and more investors began to pay attention to and participate in Bitcoin investment. The popularity of Bitcoin as a search keyword in search engines increased sharply, and the breadth and depth of cryptocurrency expanded explosively in a very short time. Facebook's Libra white paper focused people's attention on digital cryptocurrency to a higher level. Major monetary authorities and central banks regard it as a core research topic. The US Federal Reserve, the 
European Central Bank, the people's Bank of China and other institutions have all paid close attention to cryptocurrencies.

Reviewing the history and prices of cryptocurrencies represented by Bitcoin is of great significance for understanding the specific attributes of cryptocurrency. The aim of this research is to identify the role of cryptocurrencies represented by Bitcoin in the economic system and the financial market and to find the factors determine the price volatility of Bitcoin. By comparing the dynamic characteristics of the price change of cryptocurrency with that of other traditional assets (such as gold, oil, stocks, bonds, etc.), we can more accurately answer the question whether Bitcoin is a safe haven asset, digital gold, or a speculative tool. At the same time, we also investigated the dynamic correlation between Bitcoin and monetary indicators, which can help us understand the monetary attribute of Bitcoin and its relationship with real economic activities. Therefore, this study has strong theoretical and practical significance, and it is also the frontier of research in the field of monetary finance. The main content of this paper is divided into six parts: after the introduction, the theoretical analysis is discussed in part 2 . The background and existing research of Bitcoin price is summarized in part 3. The fourth part introduces the models in details; The description of the data samples used in this study is placed in part 5, which also includes the results of empirical analysis; The final part concludes the article.

\section{Theoretical approach to money and cryptocurrency}

With the continuous updating of payment technology, a large number of transactions today are completed by electronic payment (EP) system, especially in the fields of business activities, large amount payment and e-shopping. The use frequency and transaction amount of physical currency, including coins and notes, are decreasing day by day. In this sense, modern society has actually entered the era of electronic currency. However, e-money in the field of modern payment is still based on the traditional bank account framework: the central bank (monetary authority), as the only legal tender issuer in an economy, issues base currency to deposit financial institutions, which then use it to generate derivative currency. Different from the precious metal backed currency, modern currency itself has little value. Behind it is the national sovereign credit, so it is called fiat money. In most cases, the main composition of deposit financial institutions is commercial banks, and the issuance, derivation and flow of money are based on the bank account system. Therefore, this modern social credit money generation system is often called the "central bank-commercial bank account system". At this stage, digital money and electronic money are just the carriers of credit money, which does not change the original modern money payment framework with the central bank-commercial bank account system as the core. However, the raise of cryptocurrency has fundamentally broken through this original framework.

\subsection{System entropy decrease, information entropy increase and monetary consensus}

From a deeper perspective, regardless of traditional currency or cryptocurrency, the reason why currency becomes currency depends on the broad belief of the public. Friedman and Schwartz (1963) [1] called it "Convention". As Friedman (1992) [2] pointed out, the emergence, existence and evolution of money are rooted in this convention: the reason why people hold and use money for transactions is not because the substance as the currency carrier itself has value, but because they unanimously believe that the currency will also be accepted by others (Friedman (1992) [2] pointed out when discussing why US dollar notes can become a widely accepted trading intermediary:” . . private persons accept these pieces of paper because they are confident that others will. The pieces of green paper have value because everybody thinks they have value. Everybody thinks they have value because in everybody's experience they have had value - as is equally true for the stone money ... The United States could barely operate without a common and widely accepted medium of exchange (or at most a small number of such media); yet the existence of a common and widely accepted medium of exchange rests on a convention: our whole monetary system owes its existence to the mutual acceptance of what, from one point of view, is no more than a fiction." (P.18)). The process of the emergence and development of money is the process of establishing this "Convention" from scratch, from chaos to order in the economic system. In thermodynamics, the concept of entropy is used to measure the degree of chaos within a system: the larger (smaller) the entropy, the higher (lower) the degree of chaos of the system. It is easy to see that the process of realizing the monetary 
"Convention" in the economic system is equivalent to the process of entropy reduction of the system.

Further thinking, modern monetary theory holds that the essence of money is bookkeeping of transaction. Through the study of substitution effect, Kocherlakota (1996) [3] directly pointed out that money is the record of information (Kocherlakota (1996) clearly pointed out in the article 'money is memory' that "The main proposition is that any allocation that is feasible in an environment with money is also feasible in the same environment with memory." (P. 1) [3]). Just as Friedman (1992) [2] described stone money in Caroline Islands, transactions using money do not need to change the physical location of money, but only affect the bookkeeping of related parties. In modern economy, behind every transaction is the ledger recorded in currency. Therefore, the concept of information entropy can be used to investigate the evolution of money. According to the definition of Shannon (1948) [4], the fundamental function and value of information is to eliminate the uncertainty of people's cognition of things, and information entropy is to measure the amount of information needed to eliminate such uncertainty.

Suppose an information source has n possible states $x_{i}, i=1 \cdots n$, the probability of each state is $p\left(x_{i}\right)$, the information entropy of the corresponding information source is

$$
\mathrm{H}(\mathrm{X}):=-\sum_{i=1}^{n} p\left(x_{i}\right) \log _{b} p\left(x_{i}\right)
$$

Generally, $b=2$ is selected so that the unit of information entropy $\mathrm{H}(\mathrm{x})$ is byte. It is easy to see that the more chaotic a system is and the more information sources it contains, the higher the corresponding information entropy, which means that more information needs to be generated and saved to eliminate uncertainty to establish a "Convention". With the continuous occurrence of new transactions in the economic system, the information entropy of the system tends to increase. Accordingly, the information entropy needed to eliminate uncertainty and establish the "Convention" will continue to grow. According to the second law of thermodynamics, the entropy reduction process from disorder to order, that is, the process of meeting the continuously increasing information entropy, can only be realized by continuously doing work to the system.

In this article, we define this monetary "convention" as a new concept "monetary consensus". Further, according to the different mechanism and preconditions, we divide such monetary consensus into three categories: (1) Traditional monetary consensus; (2) Algorithm monetary consensus; (3) Hybrid monetary consensus. The current EC/EP system corresponds to the first type of monetary consensus, which is built on the basis of the central bank-commercial bank account system, financial infrastructure and taxation, laws and regulations, as shown in the left half of Figure 1. In order to realize this monetary consensus, the process of entropy reduction is the process of establishing and operating the grand financial system; The second type of currency consensus is the core foundation of the cryptocurrency (pure DC system) not issued by central bank. The mechanism to ensure the correctness of the ledger information recorded by the currency and the related system is no longer a centralized bookkeeping system, but an algorithm to automatically update the distributed bookkeeping system, as shown in the right half of Figure 1. The more transactions using cryptocurrency, the greater the total information entropy within the system. The work process required by the second law of thermodynamics to achieve consensus corresponds to the computational resources invested in running the algorithm (In the particular case of Bitcoin, such computational activity is called "mining".); The third type of currency consensus is the combination of the above two consensus. The digital legal currency issued by the central bank (Central Bank Digital Currency, CBDC) is an example of this mixed currency consensus, that is, DC/EP system: keep the original central bank commercial bank account system and related financial infrastructure at the EP end, and the DC side still follows the system and regulations of the central bank as the issuer of legal currency, and at the same time uses certain technology advantages of cryptocurrency, for example the distributed ledger technology (DLT).

Reviewing the history of money, the form of currency has experienced great changes, from clay (appeared around the 17th century BC), stone, shell and metal to the current paper money and electronic currency. As predicted by Friedman (1992) [2], digital currency will become the future of currency evolution (Friedman (1992) [2] pointed out when reviewing the development of money: "The 'something' that connects the two transactions is called money, and it has taken innumerable physical forms - from stones to feathers to tobacco to shells to copper, silver, and gold to pieces of paper and entries in ledger books. Who knows what will be the future incarnations of money? Computer bytes?'(P. 9)). At present, we are in a critical period from $\mathrm{EC} / \mathrm{EP}$ to DC/EP, and we need to conduct more in-depth research on digital currency. 

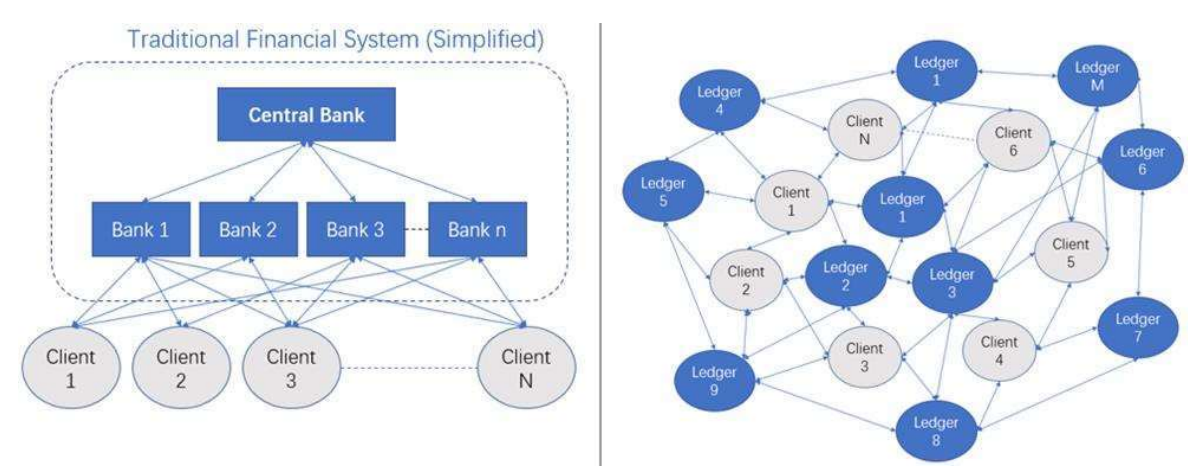

Figure 1 Currency consensus EC / EP system based on traditional financial system (left figure) and algorithm-based currency consensus distributed DC system (right figure) (Source: Own Construction)

\subsection{Pros and cons of cryptocurrencies}

Intensive debate has been occurred ever since the raise of cryptocurrencies as they are in sharp deviation to the fundamentals of modem monetary system. In the cryptocurrency system, central banks are no longer the issuer of money. Rather, private entities play active roles in providing cryptocurrencies to facilitate transactions. According to statistics, there are more than 7,800 cryptocurrencies in circulation today. The liquidity, accessibility, and thus the popularity are supported by active exchanges, in which new coins are issued and existing coins are traded, providing supply and liquidity for cryptocurrencies. In 2021, there are over 12,000 cryptocurrency exchanges (including listed and over-the-counter markets) around the world and cryptocurrencies can be used as the medium of payment in more than 100 countries.

The advocators of cryptocurrencies insist that the intrinsic nature of cryptocurrency assures the stable, or at least predictable, growth of supply [5] and thus contain the high level of inflation pressure incurred by loose monetary policy which is a "common" phenomenon in modern fiat money system after the 2008 global finance crisis. In addition, the distributed ledger technology provides a strong peer-to-peer payment mechanism that is able to work without a centralized authority and related data centers to maintain and manage huge amount of transaction data [6]. Such mechanism keeps cryptocurrencies from large scale data leakages frequently happened in the past and protect the privacy of the participants of the transaction as it is designed to be anonymous [7]. The blockchain technology also benefits the economy by lowering the cost of transactions, especially considering cross-border transactions. Empirical evidence indicates that Bitcoin lowers the transaction cost in retail foreign exchange market [8]. As summarized by the Bank of England (See the full report), distributed ledger technology that underpins cryptocurrencies "has the potential to enhance system resilience; improve the efficiency of end-to-end settlement processes and reporting, auditing and oversight; and enable greater automation.” (p. 21) The rapid growth of cryptocurrency market reflects the evolution of cryptocurrency and the increasing involvement in financial market [9].

Many institutes and researchers hold concerns and even critiques on cryptocurrency, due to its designed structure, technological details and market implications. The anonymous feature of cryptocurrency give rise to severe regulatory issues, best represented by fraud and money laundering. Cryptocurrency market is now one of the largest unregulated markets in the world. According to Foley et al. (2019) [10], approximately one-quarter of Bitcoin users are involved in illegal activity. In sum, it is estimated that around 76 billion USD illegal activities per year (roughly $46 \%$ of Bitcoin transactions) are conducted using Bitcoin. The feasibility of cryptocurrencies as the medium of payment is also challenged because of the extremely high level of price volatility [11] and considerably low capacity of processing transactions (Technical reports indicate that the maximum process capacity of DLT-based Bitcoin can hardly reach 10,000 transactions per second, while current payment systems, such as Alibaba and Visa, can handle up to 600,000 transactions per second) $[6,12]$. In a report (See the full report at https://www.bis.org/press/p201009.htm), jointly released by 7 major central banks and the Bank of International Settlement (BIS), cryptocurrencies are considered as one of the major potential threats to a coherent and well-regulated global financial system, undermining the fundamentals of domestic and international monetary system. In general, as stated by the Bank of England, "There is limited evidence of current generation of cryptoassets delivering benefits, but ... benefits may arise in the future." (p. 3) 


\subsection{Research hypotheses}

Based on theoretical analysis of the nature, technical features, and market implications of cryptocurrency, 5 hypotheses can be drawn in this research. In the first place, the acceptance of cryptocurrency as the medium of payment relies heavily on the consensus among individuals. Therefore, a positive connection between Bitcoin price and public awareness is expected. In addition, such correlation can amplify the volatility of Bitcoin price in a positive feedback circle. Such loop pushes up Bitcoin price, attracting more participants to cryptocurrency market, pursing capital appreciation rather than pure payment purpose. In this sense, Bitcoin has become an investment/speculative asset. Thus, a positive correlation between Bitcoin price with stock and bond market indices is expected. Second, as cryptocurrency develops and becomes more widely used, it tends to demonstrate positive correlation with traditional monetary and economic indices. More specifically, the more transactions used Bitcoin as payment, the higher level of connection between Bitcoin price with monetary indices, such as M0, M1, or M2. Third, due to the limited supply of Bitcoin and the technical mechanism of mining, the blockchain fundamentals of Bitcoin network, such as mining difficulty, hash value and transactions per minute, are expected to move in the same direction with Bitcoin price. Fourth, price beyond the level determined by fundamental drivers, the "fair value" of Bitcoin, can be considered as the speculative bubble, "mispricing" of Bitcoin, as shown in the work of Jia (2019) [11]. It is hypothesized that Bitcoin at current stage is more of a speculative asset rather than safe haven investments. Thus, the connection between the price of Bitcoin and of Gold is weak or even negative. Finally, certain major events may lead to the changes of Bitcoin prices, such as implementation of cryptocurrency in an economy and a revolutionary reform causes highly unstable domestic currency. These events encourage more users substitute domestic currency with cryptocurrency, thus pushing up Bitcoin price.

\section{Dynamics of Bitcoin price}

Although the concept of cryptocurrency can be traced back to a long time ago, it is generally believed that Nakamoto (2008) [13] is the first article to completely describe the architecture and practical methods of cryptocurrency. In this article, the author puts forward the theoretical basis, mathematical expression and implementation of Bitcoin. Based on the theory and method constructed by Nakamoto (2008) [13], Bitcoin was officially launched in January 2009. In the first year of online operation, the use of Bitcoin was limited to a small number of enthusiasts and had no substantive impact on the market. Until 2010, the first Bitcoin trading platforms and exchanges represented by Bitcoinmarket.com and MtGox (As once the most influential Bitcoin exchange, MtGox was officially registered and closed in February 2014. See Decker and Wattenhofer (2014) [14] and Ingram (2015) [15] for discussion and Research on the reasons for the closure of MtGox and the development of Bitcoin.) were established. The emergence of a large number of online trading platforms and exchanges (For the evolution of the exchange during the development of Bitcoin, interested readers can see https://thebitcoinnews.com/bitcoinhistory-part-6-the-first-bitcoin-exchange/.) has greatly expanded the trading convenience and liquidity of Bitcoin, attracted more institutions and individuals to join the Bitcoin system, and brought more resources and services. After such a positive cycle, the Bitcoin system continues to expand. As summarized by Kondor et al. (2014) [16], the cryptocurrency system represented by Bitcoin is an evolving network. With the increase of included nodes, the scale, convenience and influence of the network system will accelerate to enlarge, so as to absorb more nodes and resources into the system and realize positive feedback amplification. With the continuous development of Bitcoin, its price trend has also experienced great fluctuations (as shown in Figure 2), which has also attracted a large number of researchers to analyze the mechanism of Bitcoin price fluctuation.

Bouoiyour and Selmi (2017) [17] studied the main determinants of Bitcoin price using Bayesian quantile regression. The results show that hash value, geopolitical factors, macroeconomic indicators and important events are the fundamental factors that determine the price fluctuation of Bitcoin. Ciaian, Rajcaniova and Kancs (2014) [18] pointed out that the attraction of Bitcoin to investors is the main driving force of Bitcoin price. Vacha and Barunik (2012) [19] used wavelet correlation model to study the price fluctuation characteristics of energy commodity market, which is an earlier attempt using wavelet analysis method to analyze the price of investment assets. Based on similar methodology, Kristoufek and Scalas (2014) [20] analyzed the driving factors of Bitcoin price fluctuation.

Another perspective to explore the dynamics of cryptocurrency price is transparency. For financial investments, information transparency determines the quality of the underlying assets 


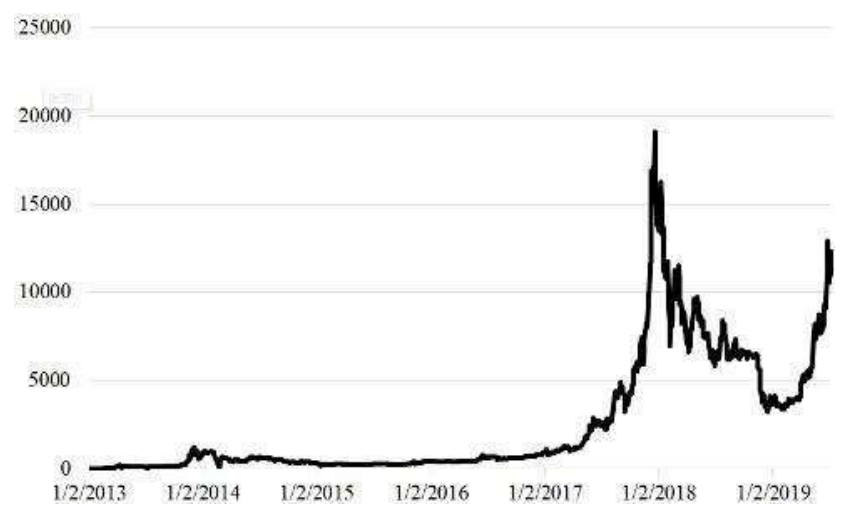

Figure 2 Bitcoin price index (USD, 20130102-20190708; Source: Bitcoinity)

and thus the intrinsic value. As shown in the work conducted by Wang et al. (2017) [21], firm value is improved by higher level of information transparency when property rights protection is in place. In the context of international trading, domestic earning transparency plays the key role in the formation of listed firm's fair value [22]. Considering the taxation and accounting standards in different regions, Ding et al. (2007) also conclude that earnings transparency is positively correlated with the fair value of listed firms. Cryptocurrencies and Bitcoin in particular can be traded around the world in various of markets and exchanges. In such sense, they are similar to listed firms. Therefore, the fair value of cryptocurrency is expected to be correlated with domestic transparency. It is a promising perspective to understand the fair value and mispricing of cryptocurrencies.

These works have achieved rich theoretical and practical results, which provides valuable inspiration and experience for this paper. On this basis, the wavelet correlation model is selected to study the time-domain and frequency-domain correlation between time series. The model of this type can accurately describe the degree of correlation, leading and lagging properties between original time-domain signals. This study also expands the scope of the original research, and introduces hedging assets, such as gold, and relevant indicators representing the international economic and political situation, such as oil price and market volatility index VIX, into the wavelet correlation analysis. In addition, we also study the relationship and difference between Bitcoin and several other major cryptocurrencies.

\section{Methodology and empirical model}

\subsection{Wavelets model}

Spectrum analysis is an effective methodology for accurate analysis of complex signals. It is widely used in electronic signal processing, electronic engineering, optics, analytical chemistry, acoustics, medicine and many other fields. In economic analysis and financial research, because the fluctuation of time series can be regarded as the result of the joint action of many factors (i.e. wavelet analysis of different spectrum), more and more researchers are committed to using wavelet analysis model in spectrum analysis to study the dynamic characteristics and mechanism of economic and financial indicators. Gallegati, Ramsey and Semmler (2011) [23] used wavelet analysis model to study the U.S. labor market, which confirmed the existence of Phillips curve. Aguiarconraria and Soares (2011) [24] used wavelet analysis model to study the fluctuation of European economic cycle.

The basis of wavelet analysis is Fourier transform, as shown in Equation (1): the original time domain signal $x(t)$ is transformed into frequency domain signal $X(f)$ by Fourier transform. This is a reversible transformation. As shown in Equation (2), the inverse Fourier transform converts the frequency domain signal into the time domain signal. In this way, the original signal can be switched between time domain and frequency domain. However, Fourier analysis cannot give the time domain and frequency domain information of the signal at the same time. Wavelet analysis model is developed to fill this gap, so that researchers can obtain both time-domain and frequency-domain information of the original time series signal at the same time.

$$
\begin{aligned}
& X(\mathrm{f})=\int_{-\infty}^{\infty} x(t) \cdot e^{-2 i \pi f t} d t \\
& x(\mathrm{t})=\int_{-\infty}^{\infty} X(f) \cdot e^{2 i \pi f t} d f
\end{aligned}
$$


As shown in Equation (3), the wavelet analysis model converts the original time series signal $x(t)$ into a function of time ( $\tau$, the position of the original signal on the time axis) and frequency (s) $-\Psi_{x}^{\varphi}(\tau, s)$ (Here s is the reciprocal of the frequency, that is, $\mathrm{s}=$ scale $=1 / \mathrm{F}$.). Here, function $\varphi$ is the wavelet function, which meets the square integrability condition $\int_{\infty}^{\infty} \varphi(t)^{2} d t<\infty$, and $\varphi^{*}$ is the conjugate of this function. Because all wavelets are derived from functions $\varphi$, it is often cited as the generating function. As can be seen from Equation (3), function $\Psi_{x}^{\varphi}(\tau, s)$ gives the similarity between the original signal at time $\tau$ and the wavelet $\varphi$ with frequency of $1 / \mathrm{s}$. By constantly changing the time value $\tau$ and $s$, the information of the original signal in the whole time period and the whole frequency period can be obtained.

$$
\Psi_{x}^{\varphi}(\tau, \mathrm{s})=\frac{1}{\sqrt{|s|}} \int_{-\infty}^{\infty} x(t) \cdot \varphi^{*}\left(\frac{t-\tau}{s}\right) d t
$$

As shown by Addison (2002) [25], there are many choices of wavelet functions $\varphi$ according to different research objects and research purposes, as long as the Admissibility Condition, $\int_{0}^{\infty} \frac{|\Psi(f)|^{2}}{f} d f<\infty$, is satisfied. In economic analysis, analytical Morlet wavelet is widely selected. Its definition is as follows:

$$
\varphi^{M}(t)=\frac{1}{\pi^{1 / 4}} e^{i \omega_{0} t} e^{-t^{2} / 2}
$$

In this equation, parameter $\omega_{0}$ represents the center frequency of wavelet. Accoding to Vacha and Barunik (2012) [19], we set $\omega_{0}=6$. By substituting Equation (4) into Equation (3), the time-domain and frequency-domain functions of the original time series $x(t), \Psi_{x}^{\varphi^{M}}(\tau, s)$, can be written using Continuous Wavelet Transform (Equation (5)):

$$
\Psi_{x}^{\varphi^{M}}(\tau, s)=\frac{1}{\sqrt{|s|}} \int_{-\infty}^{\infty} x(t) \cdot \frac{1}{\pi^{1 / 4}} e^{i \omega_{0} \frac{t-\tau}{s}} e^{-\left(\frac{t-\tau}{s}\right)^{2} / 2} d t
$$

So far, a group of time series is transformed into signals containing both time-domain and frequency-domain information through continuous wavelet transform. Due to the generating wave function $\varphi^{M}$ is a function in the complex field, so the amplitude and phase information can be obtained by studying the real part and imaginary part. In next section, we will use this property to study the correlation of two groups of time series signals after continuous wavelet transform.

\subsection{Wavelet coherence model}

Based on the wavelet model, we further construct the wavelet coherence model, and then calculate the wavelet coherence. Referring to the research of Vacha and Barunik (2012) [19] and Kristoufek and Scalas (2014) [20], the cross wavelet transform should be constructed first. Cross wavelet transform $\omega_{x y}(\tau, s)$ corresponding to two continuous wavelet transformed time series signals $x(t)$ and $y(t)$ is

$$
\omega_{x y}(\tau, \mathrm{s})=\omega_{x}(\tau, \mathrm{s}) \omega_{y}^{*}(\tau, \mathrm{s})
$$

Equation (6) indicates that the modulus of Cross Wavelet Transform $\left|\omega_{x y}(\tau, s)\right|$ captures the degree of coherence of two time series signals at each frequency s and time $\tau$. Referring to the method used by Torrence and Webster (1999), the wavelet correlation coefficient is defined as

$$
R_{x y}^{2}(\tau, \mathrm{s})=\frac{\left|S\left(\frac{1}{s} \omega_{x}(\tau, \mathrm{s}) \omega_{y}^{*}(\tau, \mathrm{s})\right)\right|^{2}}{S\left(\frac{1}{s}\left|\omega_{x}(\tau, s)\right|^{2}\right) S\left(\frac{1}{S}\left|\omega_{y}(\tau, s)\right|^{2}\right)}
$$

In Equation (7), is the smoothing operator. This equation defines the square of the correlation value of two original time series in time domain and frequency domain, with the condition of $0 \leq R_{x y}^{2}(\tau, s) \leq 1$. As described in the previous section, when the generating function in the complex domain is used for continuous wavelet transform, the phase difference between the two signals can be studied by analyzing the real and imaginary parts of the signal $\vartheta_{x y}$ (Equation $(8))$ :

$$
\vartheta_{x y}=\tan ^{-1}\left(\frac{\operatorname{Im}\left\{S\left(\frac{1}{S} \omega_{x y}(\tau, \mathrm{s})\right)\right\}}{\operatorname{Re}\left\{S\left(\frac{1}{S} \omega_{x y}(\tau, \mathrm{s})\right)\right\}}\right)
$$

The functions $\operatorname{Re}\{\cdot\}$ and $\operatorname{Im}\{\cdot\}$ return the real part and imaginary part of the corresponding value, respectively. In empirical analysis, the phase difference is represented by an arrow. When the arrow points to the right (left), it indicates that the two groups of signals are positively correlated (negatively correlated) in this area; When the arrow is up (down), it indicates that the first group of signals leads (lags) the second group of signals in this area. 


\section{Data and empirical analysis}

\subsection{Data}

To carry out empirical analysis, we need sufficient data samples. In order to study the dynamic characteristics of Bitcoin price fluctuations, especially compared with other assets, we collected six groups of data information sets:

1) Bitcoin specific data, including price, bid ask spread, price volatility, trading volume, hash rate, mining difficulty, trading volume per minute, etc;

2) Typical asset prices, including gold, oil, stock market index and bond yield;

3) Variables characterizing economic situation and market environment: dollar index and market volatility index (VIX);

4) Traditional monetary statistics, the year-on-year growth rates of US dollar M0, M1, and M2 are used in this article;

5) Public attention to Bitcoin, mainly includes the changes in the popularity in Google search using keyword "Bitcoin" in a certain region;

6) Time series of price of other digital cryptocurrencies. Considering the data availability and market influence, this article includes Ripple (XRP), Ethereum (ETH), Stella (STE) and Litecoin (LITE) with Bitcoin to study the similarities and differences of the price trajectory of cryptocurrencies.

The first group of indicators capture the unique endogenous attributes of Bitcoin. Bitcoin index comes from Bitcointy (https://data.bitcoinity.org/). Comprehensive index based on the prices of Bit-x, Bitbay, Bitfinex, Bitstamp, cex.io, CoinBase, exmo, Gemini, itbit, Kraken and other platforms; Similarly, the bid ask spread and price volatility are calculated by calculating the average value of the corresponding data in the above exchanges; The trading volume is obtained by summing up the trading volumes of all above exchanges; Bitcointy gives the hash value information of antpool, btc.top, BTCC pool, bitclub network, bitfury, bitminter, bitcoin.com, f2pool, Kano ckpool, slushpool, solo ckpool, viabtc and other platforms. Based on this, we get the comprehensive aggregate hash value; Bitcointy also provides daily data on the difficulty of Bitcoin mining.

The asset price index in the second group, market volatility index and US dollar index in the third group can be obtained through public data sources. In this article, we use Yahoo Finance and CEIC database. Among them, the gold price is the London Bullion Market Association gold price (AM), and oil price the West Texas Intermediate Cushing crude oil spot price. In this paper, the CBOT volatility index (VIX) is used to characterize the market volatility index. The larger (smaller) the VIX value, the stronger (weaker) the investor's expectation of future market volatility, and the greater (smaller) the market panic.

Bitcoin is designed to function as a currency to facilitate various transactions. Does it already have such a function or potential? A good tool is to measure its dynamic relationship with traditional currencies. In this paper, we use the M1 growth rate of US dollar to represent the traditional currency, and the data source is CEIC database. At the same time, since M1 is highly correlated with the activity of real economic activities, observing the growth rate of Bitcoin and $\mathrm{M} 1$ can also measure the dynamic relationship between Bitcoin price and macroeconomic activities from the monetary level.

In order to measure the public attention of Bitcoin, this study uses Google Statistical heat index data (https://trends.google.com) of keyword "Bitcoin". In order to more accurately describe the regional differences and the impact of certain countries, we used the search heat data of the world, the United States, India and Venezuela.

Finally, in order to study the dynamic relationship between the price fluctuation of Bitcoin and other major digital cryptocurrencies, we extract the price time series of four major digital cryptocurrencies in the market. Considering that some cryptocurrencies have been actively traded for very short period of time, we select four cryptocurrencies: Ripple (XRP), Ethereum (ETH), Stella (STE) and Litecoin (LITE). The data source for the daily price of these four cryptocurrencies is coindesk (https://www.coindesk.com).

\subsection{Empirical analysis}

The wavelet coherence model introduced in the research method part is used to empirically analyze the above data, and the wavelet coherence graph can be obtained. The correlation strength of the two signals in the specified area (time is abscissa and frequency ordinate) can be read from the wavelet correlation diagram. The higher (lower) the correlation between the two signals in a certain area of the graph, the hotter (colder) the corresponding color. Referring to the research of Vacha and Barunik (2012) [19], in economic field, generally only the fluctuation 
correlation within one year of the cycle is considered. Therefore, this study limits the ordinate range to 256 days (close to the working day time in a year). The meaning of the arrow in the wavelet correlation diagram is exactly the same as that described in the research methods section.

The debate on Bitcoin attribute and holding motivation is the main concern of the market and academia. On the one hand, many supporters of blockchain technology and cryptocurrency, especially those of Bitcoin, believe that Bitcoin is digital gold (In a sense, Bitcoin and gold do have similarities. The most important similarity is that Bitcoin and gold are limited supply growth). in the information society (refer to Popper's (2015) [26] research on the development of Bitcoin), which is a new digital currency and digital asset; On the other hand, with the rapid expansion of the cryptocurrency market represented by Bitcoin, more and more individual and institutional investors regard it as an important investment asset and occupy an important position in the portfolio; In addition, due to the extreme volatility of Bitcoin price in the short term, a large number of market participants and researchers believe that Bitcoin is a speculative asset. In order to answer a series of questions about what the attribute of Bitcoin is and why investors hold Bitcoin, we first analyze the monetary attributes of Bitcoin.

Bitcoin is designed to facilitate payment and perform the main functions of traditional currency. In terms of payment means and transaction media functions of currency, cryptocurrency has a substitution effect on traditional currency. The more transactions using digital currency, the greater the impact on the currency in traditional circulation (i.e. M0). At the same time, due to the relatively fixed supply, the price of Bitcoin tends to rise over time. From this point of view, the price of cryptocurrency has a negative correlation with the traditional currency in circulation. In terms of the value storage function of money, with the increase of economic activity and social wealth, the value stored in traditional money and digital money will increase. Therefore, there is a positive correlation between Bitcoin price and the growth rate of traditional money (M1 and M2). The differential relationship between Bitcoin price and the growth rate of traditional currencies at different levels can be clearly observed in Figure 3 and 4: since the end of 2017, Bitcoin has a high negative correlation with the growth rate of US dollar M0 and a high positive correlation with the growth rate of US dollar M1, which is reflected in the wavelet coherence graph, that is, there are large red areas in the medium and long-term frequency band. The close correlation between the price of Bitcoin and the growth of M0 and M1 also shows that Bitcoin is rapidly entering the real economy. The substitution effect on M0 is obvious. It can also be found from Figure 4 that the relationship between Bitcoin price and US dollar M2 growth is not as obvious as M0 and M1, which also shows that the replacement of traditional currency by privately issued cryptocurrency represented by Bitcoin is still at the development stage. Using the concept of currency consensus defined in the second part of this paper, it can be considered that the cryptocurrency issued by the non-central bank entities is in the second type of currency consensus, that is, the establishment stage of algorithm-based currency consensus.
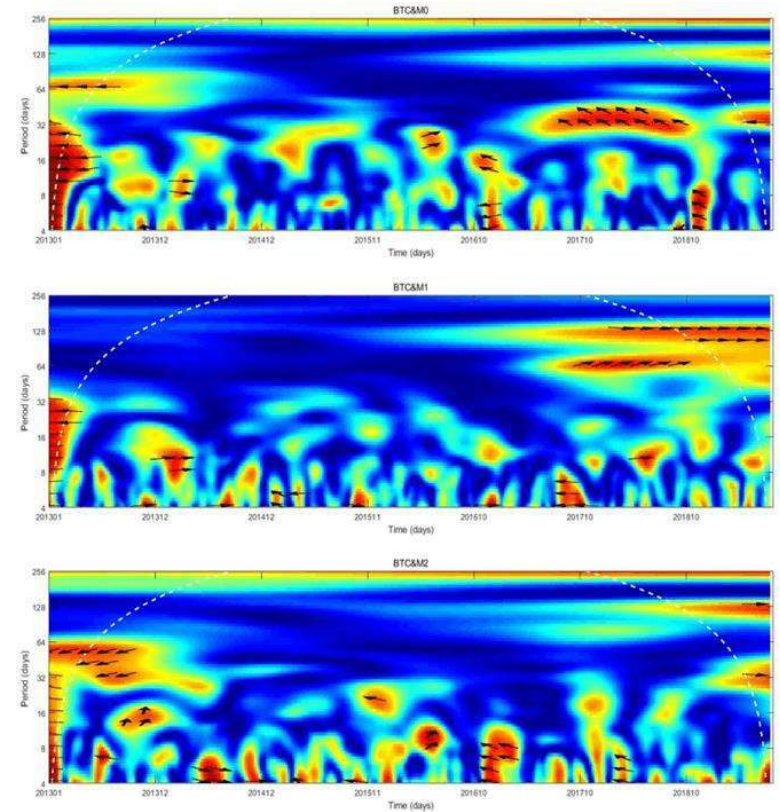

Figure 3 Coherence distribution between Bitcoin price and dollar growth at all levels 


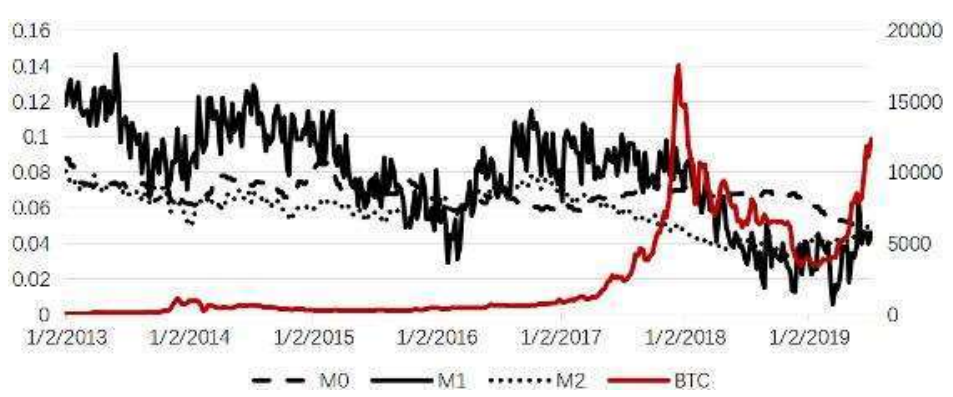

Figure 4 Bitcoin price and USD M0, M1, M2 growth rates

The functioning of Bitcoin market is an important aspect to investigate the price fluctuation mechanism of Bitcoin. In Figure 5, the correlation distribution between Bitcoin price index and Bitcoin market transaction variables is depicted. Consistent with the operating mechanism and theoretical expectation of Bitcoin, there was a high positive correlation (medium frequency to high frequency range) between Bitcoin price and price volatility and the number of transactions, from 2017 to 2018, which was a period of rapid growth of Bitcoin price. It can be seen that the increase of Bitcoin price has attracted more investors to enter the Bitcoin market, further pushing up the trading volume and price volatility. Similar to the research results of the Bitcoin currency attribute discussed earlier, Bitcoin price is highly positively correlated with the number of transactions carried out by Bitcoin (Figure 5, lower image). It once again shows that the cryptocurrency has the basic function of traditional currency as a means of payment and transaction medium, and the currency consensus of digital currency is in the formation stage.
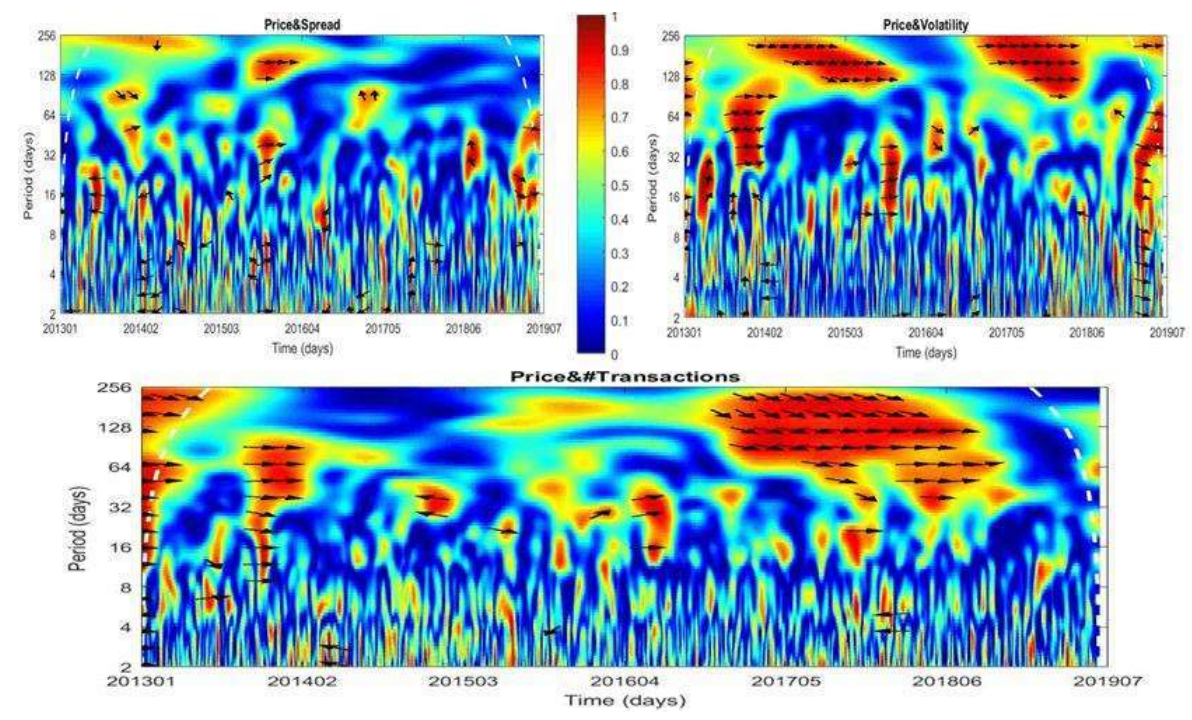

Figure 5 Coherence distribution of Bitcoin and its market transaction variables

To study the investment asset attribute of Bitcoin, we need to quantitatively study the dynamic correlation between its price and other related traditional assets and indicators. In this paper, we select three types of four typical traditional investment categories: commodity investment represented by gold and oil (at the same time, gold is also widely regarded as a safe haven asset), equity investment represented by S\&P 500 and bond investment represented by one-year US Treasury bond yield.

Figure 6 depicts the coherence between Bitcoin price and four asset prices from January 2, 2013 to July 8, 2019. The following main conclusions can be seen from the figure: (1) The correlation between Bitcoin and gold and other assets is not obvious in the short-term frequency period (the time length is less than one and a half months); (2) From 2015 to 2016, Bitcoin and crude oil prices were highly negatively correlated in the medium term (one and a half to six months), and oil prices moves ahead of Bitcoin prices. After that, the coherence weakens; (3) After 2018, the coherence between Bitcoin assets and stock market and bond market indicators has significantly increased in the medium and long-term (one and a half months to one year) band. This shows that with the rapid expansion of Bitcoin system and the increase in the number of investors, Bitcoin is becoming a potential investment asset in parallel with the stock market, 
bond market and commodity market.
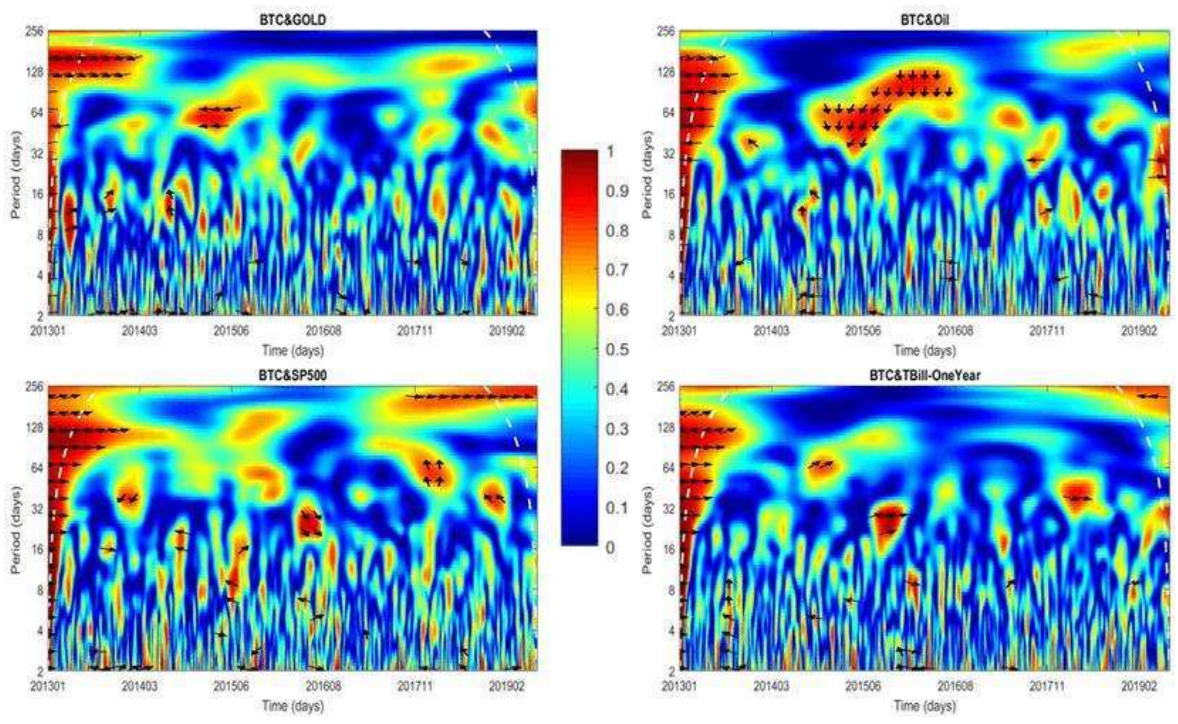

Figure 6 Coherence distribution of Bitcoin price with gold, oil, S \& P500 and US one-year treasury bond yield

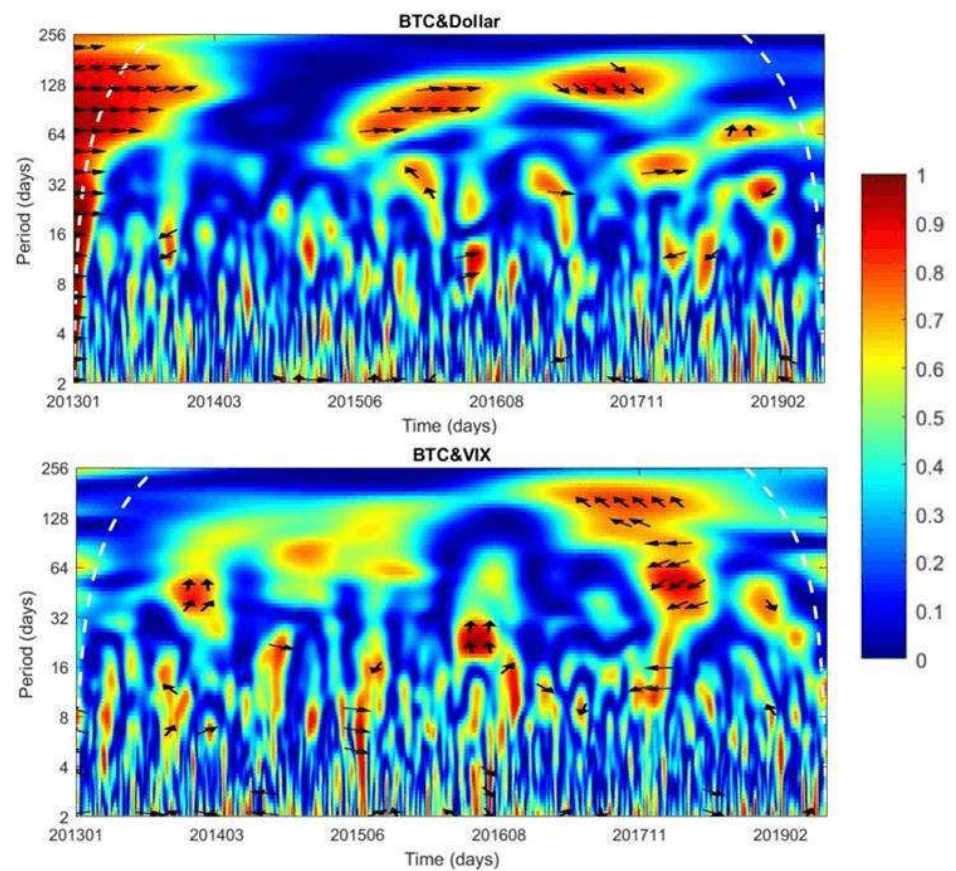

Figure 7 Coherence distribution of Bitcoin price with us dollar index and market volatility index

In Figure 7, it can be found that there is a strong positive correlation between Bitcoin price and US dollar index in the medium and long-term frequency band since 2015. The possible explanation for this result is that if Bitcoin is regarded as an investment product with substitution effect with traditional assets, the improvement of the US dollar index puts downward pressure on the price of commodities denominated in US dollars. At the same time, the economic growth of developed economies outside the United States weakens, making part of the investment funds flow from the traditional market to the Bitcoin market. It can be seen from Figure 7 that around the end of 2017, Bitcoin and market panic index VIX showed an obvious negative correlation in the medium and long-term frequency band. This can be explained by the wealth effect of US dollar assets and stock investment. The appreciation of US dollar assets and the return of stock investment increase the investable capital of investors. At the same time, the optimistic expectation of the market further promotes the investment willingness. Therefore, more funds can be invested in the Bitcoin Market. At the same time, the negative correlation 
between Bitcoin price and market panic index also shows that Bitcoin does not have strong characteristics of safe haven assets. This conclusion is consistent with the conclusion that there is no obvious correlation between Bitcoin and gold price shown in Figure 6 .

As mentioned earlier, Bitcoin is a cryptocurrency based on blockchain technology, so its price change is also related to the operation of the blockchain. In Figure 8, we summarize the distribution of the correlation between Bitcoin price and blockchain operation indicators in time domain and frequency domain. In this figure, it can be seen that in 2013, Bitcoin price is highly related to the long-term trend of blockchain operation fundamentals such as hash value, mining difficulty and transaction volume per minute. However, after that, the correlation decreased sharply. Especially during the period of Bitcoin price surge in 2017 and 2018, the correlation between Bitcoin price and blockchain fundamental data is very low. Therefore, the rapid increase in prices at this stage can be interpreted as a bubble driven by investment and speculation, rather than fundamentals.
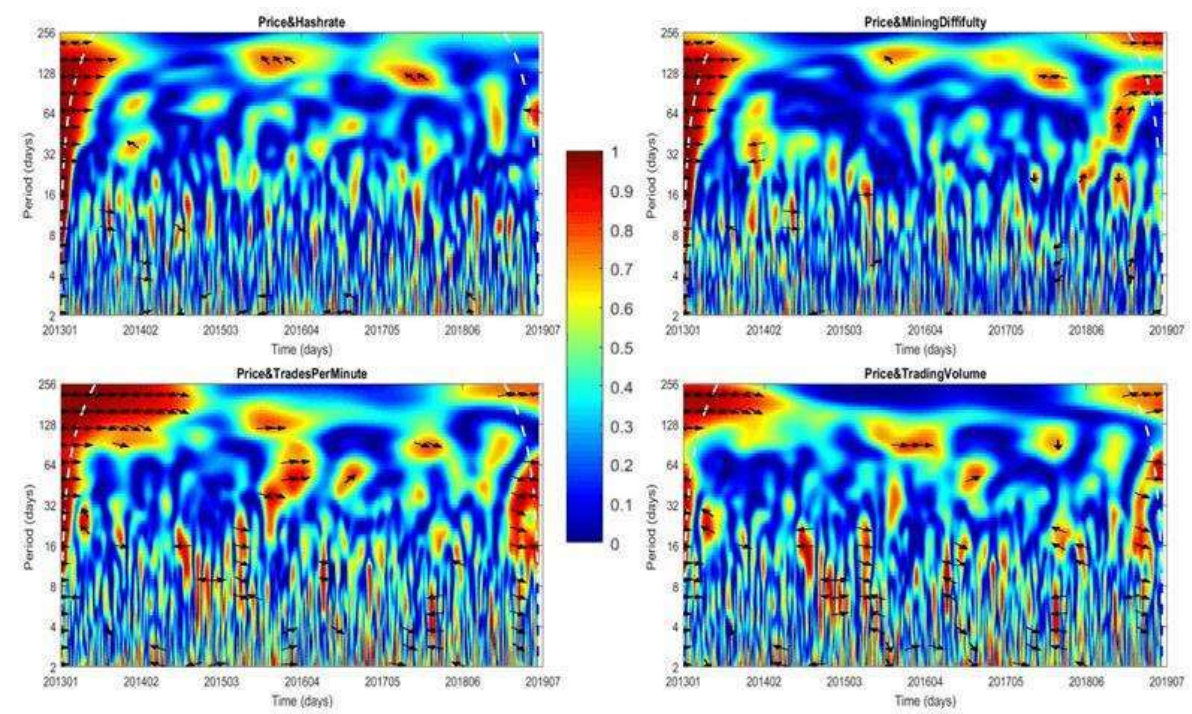

Figure 8 Coherence distribution of Bitcoin and blockchain variables

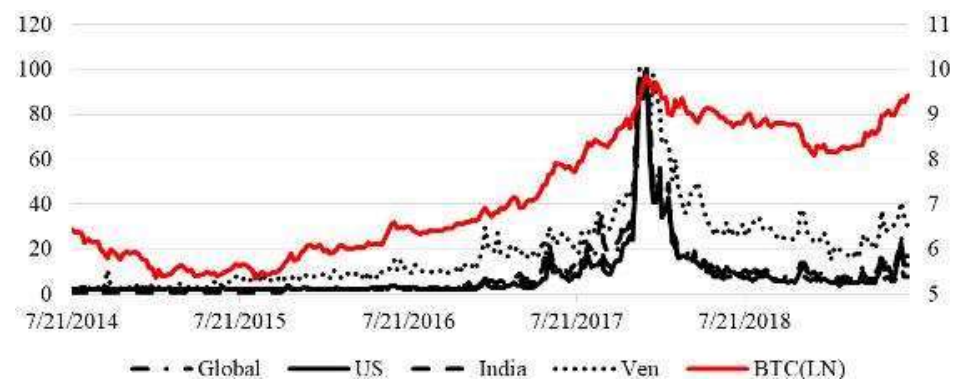

Figure 9 Bitcoin price (take the logarithm of its natural base, right axis) and changes in Google search popularity in the world (global), the United States (US), India and Venezuela (left axis)

As Bitcoin is still in the stage of development and expansion, the increase in the number of Bitcoin investors and users has played a decisive role in the trend of Bitcoin price. Therefore, the higher the attention of the public group to Bitcoin, the greater the number of potential investors and users of Bitcoin, and thus higher Bitcoin price. This trend can be clearly seen from Figure 10. Bitcoin price is highly positively correlated with the operation trend of search popularity. Referring to many previous studies, in this paper, we use the popularity of the search keyword "Bitcoin" in the search engine to characterize this index. We can clearly see this trend. Bitcoin price is highly related to the trend of search popularity. Figure 11 shows the analysis results of wavelet coherence. It can be seen that since 2015, there has been a high positive coherence between Bitcoin price and search popularity in all frequency bands, indicating that public awareness is one of the main driving factors of Bitcoin price, and the addition of new investment has increased the volatility of Bitcoin price. This conclusion is consistent with our hypothesis and empirical evidence that there is a positive correlation between Bitcoin price and 
its volatility, especially from 2017 to 2019 when Bitcoin price fluctuates sharply (as shown in Figure 7). This also shows that the Bitcoin market is still in a period of rapid expansion, and the main driving force for the growth of Bitcoin price is incremental investment. This conclusion is in good agreement with the previous research results and our expectations, and also supports the research conclusions of Ciaian, Rajcaniova and Kancs (2014) [18].

It is worth noting that for economies with extremely unstable domestic currencies (represented by India and Venezuela in this study), the correlation between search heat index and Bitcoin price in the short term is much higher than the global average and developed countries. India launched the "banknote reform" in November 2016 and abolished large Rupee banknotes; Venezuela started currency reform in December 2016 and August 2018. As shown in Figure 9 , at these time points, the Bitcoin price is highly positively correlated with the country's Google search popularity in both medium and high frequencies, also indicating that the Bitcoin price can be driven by major events, confirming our hypothesis.

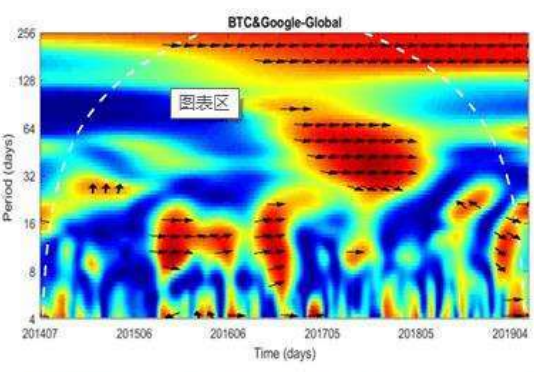

BTC\&Googletendia

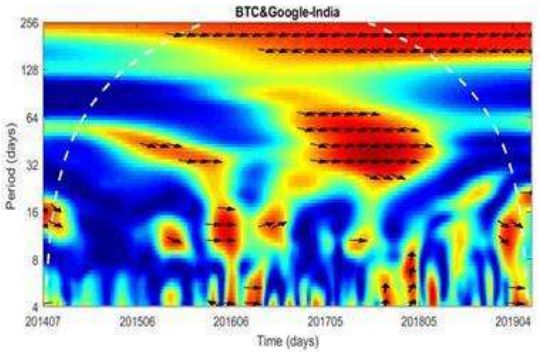

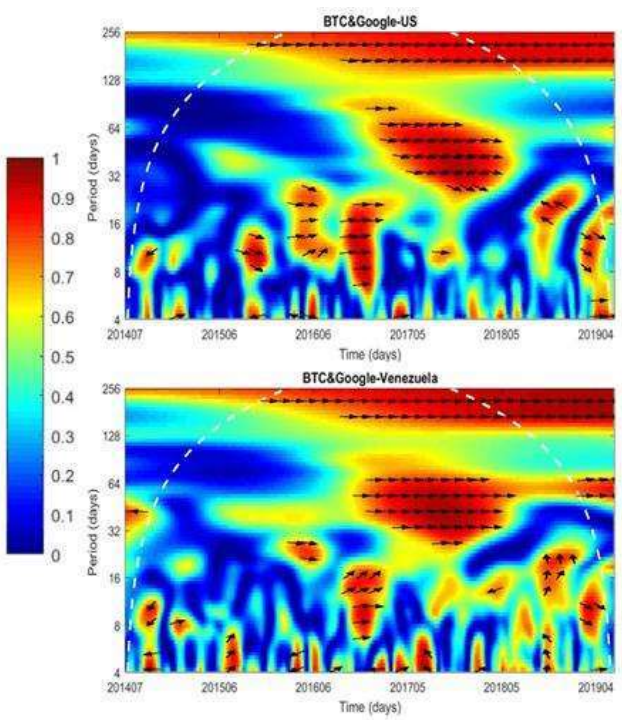

Figure 10 Coherence distribution between Bitcoin price and Google search popularity
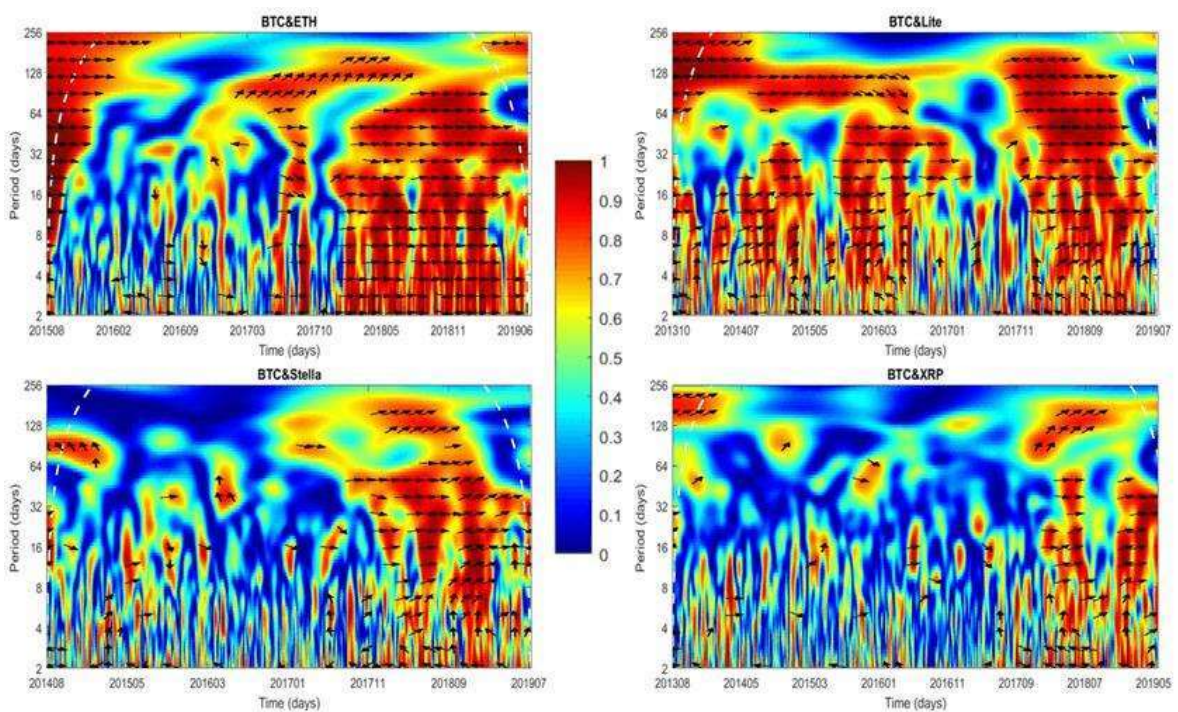

Figure 11 Distribution diagram of price fluctuation coherence between Bitcoin and other four digital cryptocurrencies

To study the dynamic relationship between the price fluctuation of Bitcoin and other major cryptocurrencies, we extracted the price trend information of four major cryptocurrencies in the market. We select four major cryptocurrencies: Ripple (XRP), Ethereum (ETH), Stella (STE) and Litecoin (LITE). Figure 10 shows the coherence distribution of price fluctuations between Bitcoin and the four cryptocurrencies. It can be seen that Bitcoin has a high positive 
correlation with Ethereum and Litecoin, while it has a low correlation with Stella and XRP. It should be emphasized that after 2018, the coherence between Bitcoin and all the four cryptocurrencies has increased significantly in almost all frequency bands, indicating that the price trend consistency of cryptocurrencies has been rapidly strengthened. With the rapid popularization of cryptocurrency represented by Bitcoin after 2018, the cryptocurrency market is moving from dispersion to concentration. Therefore, the price trajectory of each cryptocurrency has becoming more and more consistent.

\section{Conclusion and discussion}

With the development of payment system, e-money has become the main monetary carrier of various transaction activities in social production and life. The use frequency and transaction amount of real currency, including coins and notes, are decreasing day by day. In this sense, modern society has actually entered the era of electronic money and electronic payment (EC/EP). Cryptocurrency issued by private entities is committed to establishing a new monetary and financial system based on blockchain technology. This system adopts decentralized distributed processing mechanism and makes full use of existing Internet nodes and computing resources, which can greatly reduce transaction costs, effectively solve the double-spending problem and protect users' personal information. Bitcoin, as the most influential cryptocurrency and the currency with the highest market share, has attracted the attention of the market in a short time. In particular, the sharp rise in the price of Bitcoin in the past 2-3 years has attracted extensive attention, and also intensified the debate on the currency attribute and the motivation of holding cryptocurrency. In theory, by introducing entropy subtraction and information entropy of economic system, combined with monetary theory, this study puts forward the concept of "monetary consensus", and defines three types of monetary consensus. In terms of empirical analysis, by comparing the dynamic characteristics of the price change of cryptocurrency with the growth rate of traditional currencies at different levels (USD M0, M1 and M2) and the price change of other traditional assets (such as gold, oil, stocks, bonds, etc.), we can more accurately answer the question about whether Bitcoin has the attribute of currency and investment assets.

The analysis of the dynamic correlation between time series needs to calculate the dynamic relationship in time domain and frequency domain. The correlation model in wavelet analysis model just meets this demand. In this paper, the original time series signals are transformed into complex functions in time domain and frequency domain by continuous Morlet wavelet transform. At the same time, the intensity and phase angle of wavelet correlation coefficient are defined by cross wavelet transform, so that the correlation distribution of two time series signals in time domain and frequency domain can be analyzed. Empirical analysis shows good consistence with research hypotheses. In conclusion, the following theoretical and empirical findings are obtained:

Firstly, we define the essence of modern money as a new concept "monetary consensus", and divide it into three categories according to the generation mechanism and preconditions: (1) Traditional monetary consensus; (2) Algorithm monetary consensus; (3) Hybrid monetary consensus. The current EC/EP system corresponds to the first type of monetary consensus, which is based on the central bank-commercial bank account system, the construction of largescale financial infrastructure and the construction of taxation, laws and regulations. In order to realize this monetary consensus, the process of entropy reduction is the process of establishing and operating system; The second type of currency consensus is the core foundation of the cryptocurrency (pure DC system) issued by the non-central bank entities. The mechanism to ensure the correctness of the ledger information recorded by the currency is no longer a centralized accounting system, but automatically updates the distributed accounting system by relying on the algorithm. The more transactions using cryptocurrency, the greater the total information entropy within the system. The work process required by the second law of thermodynamics to achieve consensus corresponds to the computational resources invested in running the algorithm; The third type of currency consensus is the combination of the above two consensus. The digital legal currency issued by the central bank is an example of this mixed currency consensus, that is, DC/EP system: utilize the original central bank commercial bank account system and related financial infrastructure at the EP end, while the DC side still follows the system and regulations of the central bank as the issuer of legal currency, and uses certain technical features of cryptocurrency.

Secondly, since the end of 2017, Bitcoin has been highly negatively correlated with the growth rate of US dollar M0 and highly positively correlated with the growth rate of US dollar M1, indicating that Bitcoin is rapidly entering the real economy, has obvious substitution effect on M0 and has considerable value storage function. This finding verifies our hypothesis that 
as cryptocurrency becomes more widely used in transaction, its connection with traditional monetary indices enhances as well. The relationship between Bitcoin price and the growth rate of US dollar M2 is not as obvious as M0 and M1, which also shows that the replacement of traditional currency by cryptocurrency issued by non-central bank entities represented by Bitcoin is still in the development period. It can be considered that the cryptocurrency issued by non-central bank entities is in the stage of establishing currency consensus based on algorithm.

In the third place, the correlation between Bitcoin and assets such as gold is not obvious in the short term (less than one and a half months). However, after 2018, the correlation between Bitcoin assets and stock market and bond market indicators has significantly increased in the medium and long-term band. This shows that with the rapid expansion of Bitcoin system and the increase in the number of investors, Bitcoin, as our first hypothesis predicted, has becoming a potential investment asset in parallel with the stock market, bond market and commodity market;

Fourth, since 2015, there has been a strong positive correlation between Bitcoin price and US dollar index in the medium and long-term frequency band. Around the end of 2017, Bitcoin and market VIX showed an obvious negative correlation in the medium and long-term frequency band. This can be explained by the wealth effect of US dollar assets and stock investment. The appreciation of US dollar assets and the return of stock investment increase investable capital. At the same time, the optimistic expectation of the market further promotes the investment willingness. Therefore, more funds can and tend to be invested in Bitcoin Market. At the same time, the negative correlation between Bitcoin price and market panic index also shows that Bitcoin does not have strong characteristics of safe haven assets to a certain extent, further supporting the hypothesis that Bitcoin is of speculative/investment asset rather than safe haven asset;

Fifth, the increase in the number of Bitcoin investors and users has played a decisive role in the trend of Bitcoin price: since 2015, there has been a high positive correlation between Bitcoin price and search popularity in all frequency bands. The public popularity of Bitcoin is one of the main driving factors of its price, and the addition of new investment has amplified price volatility. This finding verifies our hypothesis that Bitcoin price is positively correlated with public awareness of Bitcoin. It is worth noting that for economies with extremely unstable currencies (such as India and Venezuela), the search heat index has a far higher correlation with Bitcoin price in the short-term than the global average and developed countries. At the time of currency reform in India and Venezuela, the high-frequency positive correlation between Bitcoin price and search popularity in that country has increased rapidly in the short-term, which also reflects that the fluctuation of Bitcoin price is affected by major events. This is consistent with our final hypothesis that major events may give rise to the fluctuations of Bitcoin price;

Sixth, during the period of rapid growth of Bitcoin price in 2017 and 2018, the correlation between Bitcoin price and blockchain fundamentals is low. The rapid increase in prices at this stage can be interpreted as a bubble driven by investment and speculation rather than a price growth supported by fundamentals. Therefore, Bitcoin assets have strong speculative tool attributes at present. This is consistent with our fourth hypothesis and the findings of Jia (2019) [11];

Seventh, with the accelerated popularity of cryptocurrencies represented by Bitcoin after 2018, the cryptocurrency market is moving from dispersion to concentration. Therefore, the price indices of cryptocurrencies converge.

In short, the theoretical contribution of this research is the concept of "monetary consensus" with three types, based on reviewing the evolution of money. By applying such concept, we can identify and evaluate the stage and trend of monetary system, providing valuable information for policy makers and system designers. At present, China is in the key stage of moving from EC/EP to DC/EP. In order to better realize the safe and stable substitution of CBDC for M0, we need to realize the monetary consensus of CBDC, combing the legal tender consensus and the algorithm consensus. The empirical analysis shows good consistence with theoretical hypotheses in terms of the connections between Bitcoin and market indices, quantitively identify the monetary and investment attributes of Bitcoin. These findings help market participates and researchers better understand the dynamics of Bitcoin prices and the motives behind the demand for Bitcoin. The limitation of this research is that it focuses on the monetary and investment attributes of Bitcoin, by investigating the time and frequency correlation between Bitcoin and market indices. The next step is to analyze the dynamics of Bitcoin, or cryptocurrencies in general, in the context of economic and financial system, building dynamic models explicitly contain cryptocurrency to capture a more comprehensive image of cryptocurrency. 


\section{Acknowledgements}

This study is supported by the National Postdoctoral International Exchange Program Recruitment Project, China Postdoctoral Science Foundation. Any opinions expressed are those of the authors and not those of the institutes the authors are affiliated to.

\section{References}

[1] Friedman M and Schwartz J. A Monetary History of the United States, 1867-1960. NBER Books, 1963, 70(1): 512-523. https://doi.org/10.2307/1842177

[2] Friedman M. Money mischief: episodes in monetary history. Harcourt brace Jovanovich, Publishers, 1992.

[3] Kocherlakota R. Money Is Memory. Staff Report, 1996, 81(2): 232-251. https://doi.org/10.21034/sr.218

[4] Shannon E. A mathematical theory of communication. Bell Labs Technical Journal, 1948, 27(4) :379-423. https://doi.org/10.1002/j.1538-7305.1948.tb01338.x

[5] Iwamura M, Kitamura Y, Matsumoto T, et al. Can We Stabilize the Price of a Cryptocurrency?: Understanding the Design of Bitcoin and Its Potential to Compete with Central Bank Money. Discussion Paper Series, 2014, 617: 1-31. https://doi.org/10.2139/ssrn.2519367

[6] Mukhopadhyay U, Skjellum A, Hambolu O, et al. A brief survey of Cryptocurrency systems, 2016 14th Annual Conference on Privacy, Security and Trust (PST). IEEE, 2016. https://doi.org/10.1109/PST.2016.7906988

[7] Peters G, Panayi E and Chapelle A. Trends in Crypto-Currencies and Blockchain Technologies: A Monetary Theory and Regulation Perspective. Social Science Electronic Publishing, 2015, 3: 1-25. https://doi.org/10.2139/ssrn.2646618

[8] Kim T. On the transaction cost of Bitcoin. Finance Research Letters, 2017, 23: 300-305. https://doi.org/10.1016/j.frl.2017.07.014

[9] Elbahrawy A, Alessandretti L, Kandler A, et al. Evolutionary dynamics of the cryptocurrency market Royal Society Open Science, 2017, 4(11): 170623. https://doi.org/10.1098/rsos.170623

[10] Foley S, Karlsen JR and Putniņš TJ. Sex, drugs, and Bitcoin: How much illegal activity is financed through cryptocurrencies? Review of Financial Studies, 2019, 32(5): 1798-1853. https://doi.org/10.1093/rfs/hhz015

[11] Jia L. Price Fluctuations of the Decentralized Cryptocurrency-PCA/DFM Analysis of the Bitcoin Price. Journal of Financial Development Research, 2019, 11: 53-57.

[12] Dwyer GP. The economics of Bitcoin and similar private digital currencies. Journal of Financial Stability, 2015, 17: 81-91. https://doi.org/10.1016/j.jfs.2014.11.006

[13] Nakamoto S. Bitcoin: A Peer-to-Peer Electronic Cash System, 2008. https://bitcoin.org/en/bitcoin-paper

[14] Decker C and Wattenhofer R. Bitcoin Transaction Malleability and MtGox. In: Kutyłowski M., Vaidya J. (eds) Computer Security - ESORICS 2014. ESORICS 2014. Lecture Notes in Computer Science, vol 8713. Springer, Cham, 2014. https://doi.org/10.1007/978-3-319-11212-1_18

[15] Ingram C. "A bad apple went away": Exploring resilience among Bitcoin entrepreneurs, European Conference on Information Systems, 2015.

[16] Kondor D, Posfai M, Csabai I, et al. Do the Rich Get Richer? An Empirical Analysis of the Bitcoin Transaction Network. Plos One, 2014, 9(2): e97205. https://doi.org/10.1371/journal.pone.0086197

[17] Bouoiyour J and Selmi R. The Bitcoin price formation: Beyond the fundamental sources. Papers, 2017, 1-28. https://doi.org/10.13140/RG.2.2.23880.32000

[18] Ciaian P, Rajcaniova M and Kancs D. The Economics of BitCoin Price Formation. EERI Research Paper Series, 2014, 48(19): 1799-1815. https://doi.org/10.1080/00036846.2015.1109038

[19] Vacha L and Barunik J. Co-movement of energy commodities revisited: Evidence from wavelet coherence analysis. Energy Economics, 2012, 34(1): 241-247. https://doi.org/10.1016/j.eneco.2011.10.007

[20] Kristoufek L and Scalas E. What Are the Main Drivers of the Bitcoin Price? Evidence from Wavelet Coherence Analysis. Plos One, 2014, 10(4): e0123923. https://doi.org/10.1371/journal.pone.0123923

[21] Wang HJ, Li MM and Xu QF. Property Rights Protection,Information Transparency and Firm ValueEmpirical Evidence from Shenzhen A-share Listed Firms. Finance Research, 2017. 
[22] Delimatsis P. International Trade in Services and Domestic Regulations: Necessity, Transparency, and Regulatory Diversity. Oup Catalogue, 2009, 12(2): 3165-3173.

https://doi.org/10.1093/jiel/jgp004

[23] Gallegati M, Ramsey B and Semmler W. The US Wage Phillips Curve across Frequencies and over Time. Oxford Bulletin of Economics and Statistics, 2011, 73(4): 489-508. https://doi.org/10.1111/j.1468-0084.2010.00624.x

[24] Aguiarconraria S and Soares J. Business Cycle Synchronization and the Euro: A Wavelet Analysis. Journal of Macroeconomics, 2011, 33(3): 477-489. https://doi.org/10.1016/j.jmacro.2011.02.005

[25] Addison PS. The Illustrated Wavelet Transform Handbook: Introductory Theory and Applications in Science. Science Engineering Medicine \& Finance Institute of Physics Publishing, 2002. https://doi.org/10.1887/0750306920

[26] Popper N. Digital Gold: The Untold Story of Bitcoin. Penguin Books Ltd, ISBN: 9780241180990 , 2016 